\title{
Cardiac Calcified Amorphous Tumor in the Left Atrium: A Case Report
}

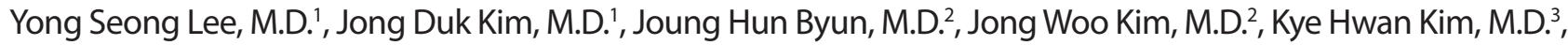 \\ Ji Min Na, M.D. ${ }^{4}$, Hyun Oh Park, M.D. ${ }^{1}$
}

'Department of Thoracic and Cardiovascular Surgery, Gyeongsang National University Hospital, Gyeongsang National University College of Medicine, Jinju; ${ }^{2}$ Department of Thoracic and Cardiovascular Surgery, Gyeongsang National University Changwon Hospital, Gyeongsang National University College of Medicine, Changwon; ${ }^{3}$ Division of Cardiology, Department of Internal Medicine and ${ }^{4}$ Department of Pathology, Gyeongsang National University Hospital, Gyeongsang National University College of Medicine, Jinju, Korea

\section{ARTICLE INFO}

Received July 29, 2021

Revised October 8, 2021

Accepted October 8, 2021

Corresponding author

Hyun Oh Park

Tel 82-55-750-8124

Fax 82-55-753-8138

E-mail cspark@gnuh.co.kr

ORCID

https://orcid.org/0000-0003-1302-6456
A cardiac calcified amorphous tumor (CAT) is a rare non-neoplastic mass of the heart with histological characteristics comprising calcification and amorphous fibrous material. Little is known regarding the incidence, symptoms, and therapeutic strategies for CAT. Echocardiography and computed tomography were performed on a 48-year-old man who had a cardiac mass that was accidentally discovered on admission to a local hospital for a fracture related to a trauma that occurred 1 month prior. After surgery, a histological examination resulted in the diagnosis of a cardiac CAT. The patient was discharged without postoperative complications on the 12th day after surgery.

Keywords: Heart, Neoplasms, Physiologic calcification, Case report

\section{Case report}

A 48-year-old male patient visited Gyeongsang National University Hospital with an intracavitary cardiac mass that was accidentally discovered while he was admitted to a local hospital for a trimalleolar fracture related to trauma that occurred 1 month prior. The patient was a non-smoker and was taking drugs for cirrhosis and epilepsy; there was no other pertinent medical history. Upon examination, he presented with normal vital signs, and cardiac murmur was absent. Laboratory tests revealed 3,360 leukocytes $/ \mathrm{mm}^{3}$ with the normal formula, hemoglobin of $12.0 \mathrm{~g} / \mathrm{dL}, 78,000$ platelets $/ \mathrm{mm}^{3}$, a creatinine level of $1.18 \mathrm{mg} / \mathrm{dL}$, a C-reactive protein level of $7.7 \mathrm{mg} / \mathrm{L}$, and cardiac biomarkers in the normal range. No bacteria were identified in the blood culture. A chest radiograph revealed a lesion suspected of being an intracardiac mass (Fig. 1A). Computed tomography (CT) and echocardiography were performed for a more
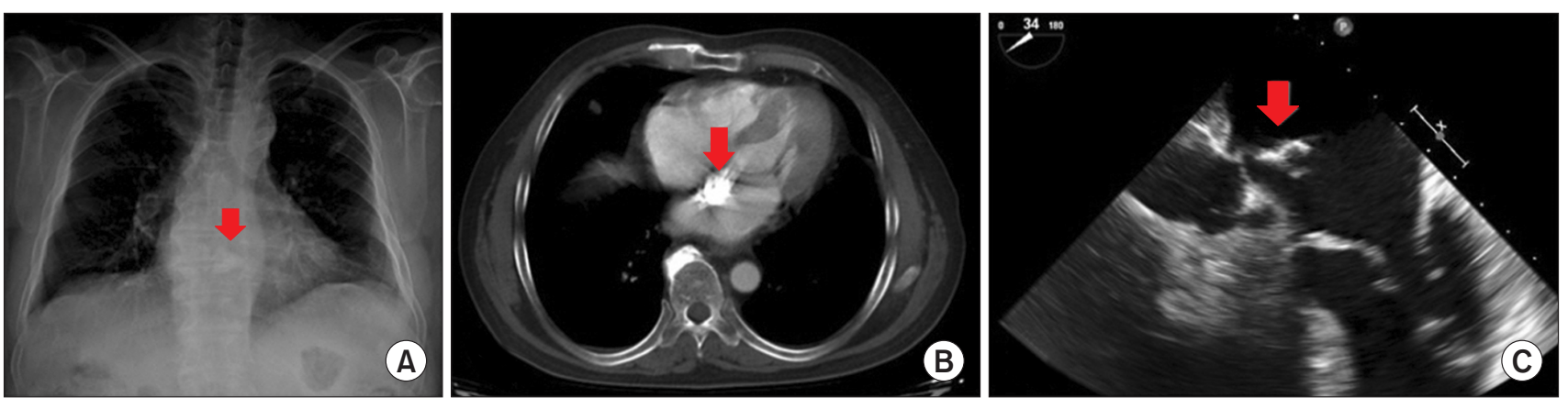

Fig. 1. (A) Chest radiography showing an intracardiac mass lesion (arrow). (B) Computed tomography showing a hyperdense calcified mass within the left atrium (arrow). (C) Echocardiography showing a large calcified mass attached to the interatrial septum superior to the mitral valve (arrow). 
precise assessment. CT of the chest detected a $2.8 \times 2.0 \mathrm{~cm}$ calcified mass in the left atrium (Fig. 1B). Echocardiography revealed a mobile mass attached to the interatrial septum superior to the posterior mitral valve leaflet (Fig. 1C). Since the mass was mobile and the risk of embolization was high, we decided to remove the mass surgically. Arterial cannulation for the aorta and venous cannulation for the superior and inferior vena cava were employed for cardiopulmonary bypass (CPB). Moderate systemic hypothermia was attained with $\mathrm{CPB}$. The ascending aorta was cross-clamped and a cardioplegic histidine-tryptophan-ketoglutarate solution (Dr. Franz Kohler Chemie GmbH, Bensheim, Germany) was administered via a root cannula in the ascending aorta. The tumor-invaded portion of the atrial septum was completely resected and reconstructed with autologous pericardium. A $3.2 \times 2.0 \times 2.0 \mathrm{~cm}$ yellow and ivory-colored hard calcified mass (Fig. 2) attached to the atrial septum without adhesion to the mitral annulus was removed without any surgical complications. The total operation time was 2.5 hours, $\mathrm{CPB}$ time was 88 minutes, and aortic cross-clamping time was 52 minutes.

Histopathological examination of the mass revealed diffuse calcification in the stroma, with degenerated amorphous debris, fibrous material, and indistinguishable myxoma or malignant cells (Fig. 3); the patient was diagnosed with a calcified amorphous tumor (CAT) of the heart. On hospital day 12, the patient was discharged without any postoperative complications. The Human Research Ethics Committee of Gyeongsang National University Hospital provided a waiver considering that approval is not necessary for a single case report. The patient provided written informed consent for publication of the clinical details and images.

\section{Discussion}

Cardiac CAT is a rare non-neoplastic mass of the heart
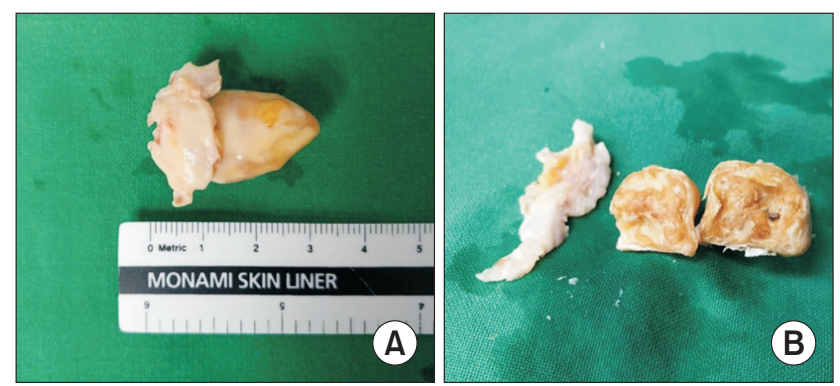

Fig. 2. (A, B) A $3.2 \times 2.0 \times 2.0 \mathrm{~cm}$ yellow and ivory-colored hard calcified mass. with histological characteristics comprising calcification and an amorphous fibrous material [1]. The term CAT was first proposed by Reynolds et al. [2] in 1997 based on the clinical and microscopic appearance of 11 patients at the Mayo Clinic. Although the possibilities of chronic inflammation, hypercoagulability, organized calcific mural thrombus, and abnormal calcium and phosphate metabolism have been mentioned, the etiology of CAT has not been clearly elucidated [3]. Mitral valve disease (31\%) is the most commonly associated condition, followed by endstage renal disease $(21 \%)$, mitral annulus calcification (MAC) (14\%), diabetes (14\%), and coronary disease (12\%) [1]. CAT can occur in all chambers of the heart, especially around the mitral valve (36\%) [1]. The most common symptoms are dyspnea (45\%), syncope (21\%), pulmonary embolism (8\%), and systemic embolism (12\%) [1,3]. Although ventricular arrhythmias have been reported, their causal relationship with CAT has not yet been established [4]. The CAT growth rate is not clear; however, it could be relatively fast for MAC-related CAT [4].

CT and echocardiography can help diagnose CAT by detecting calcified masses in the heart [5]. However, it is difficult to clearly differentiate between CAT and osteosarcoma, leiomyosarcoma, calcified myxoma, or vegetation using only cardiac imaging [5]. Reynolds et al. [2] defined CAT as a non-neoplastic cardiac mass consisting of a calcified nodule, fibrous material, and amorphous debris; surgical resection is required for diagnosis. Surgical removal is relatively safe [1]; however, there have been reports of patients who have died while removing the ventricular myocardium [6] and relapsed after incomplete resection [7]. Therefore, complete resection is generally proposed as a therapeutic strategy; however, further studies are needed in patients with diffuse myocardial involvement of CAT [1].

Little is known regarding the incidence, symptoms, and therapeutic strategies for CAT [5]. Prospective studies are
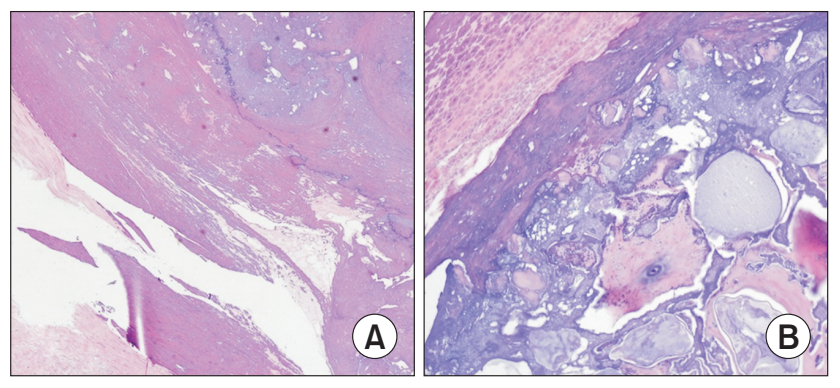

Fig. 3. (A, B) Histologic image of a hematoxylin and eosin-stained calcified amorphous tumor $(A: \times 100 ; B: \times 200)$ with diffuse calcification on the background of degenerated amorphous debris and fibrous material. 
needed to clearly understand its characteristics. Therefore, we believe that this report provides meaningful clinical data to help understand the characteristics of CAT. We suggest that CAT should be considered if a mass shows distinct characteristics from those of general myxoma in a preoperative examination.

\section{Conflict of interest}

No potential conflict of interest relevant to this article was reported.

\section{ORCID}

Yong Seong Lee: https://orcid.org/0000-0003-0789-3916 Jong Duk Kim: https://orcid.org/0000-0003-0268-1674 Joung Hun Byun: https://orcid.org/0000-0003-1909-664X Jong Woo Kim: https://orcid.org/0000-0003-2578-9821 Kye Hwan Kim: https://orcid.org/0000-0001-5234-1251 Ji Min Na: https://orcid.org/0000-0003-4330-6598 Hyun Oh Park: https://orcid.org/0000-0003-1302-6456

\section{References}

1. De Hemptinne Q, de Canniere D, Vandenbossche JL, Unger P. Cardiac calcified amorphous tumor: a systematic review of the literature. Int J Cardiol Heart Vasc 2015;7:1-5.

2. Reynolds C, Tazelaar HD, Edwards WD. Calcified amorphous tumor of the heart (cardiac CAT). Hum Pathol 1997;28:601-6.

3. Shah AC, Marcoff L, Talati S, et al. A rare beast: cardiac calcified amorphous tumor. CASE (Phila) 2018;2:139-41.

4. Kubota H, Fujioka Y, Yoshino H, et al. Cardiac swinging calcified amorphous tumors in end-stage renal failure patients. Ann Thorac Surg 2010;90:1692-4.

5. Miller DV, Tazelaar HD. Cardiovascular pseudoneoplasms. Arch Pathol Lab Med 2010;134:362-8.

6. Lewin M, Nazarian S, Marine JE, Yuh DD, Argani P, Halushka MK. Fatal outcome of a calcified amorphous tumor of the heart (cardiac CAT). Cardiovasc Pathol 2006;15:299-302.

7. Fealey ME, Edwards WD, Reynolds CA, Pellikka PA, Dearani JA. Recurrent cardiac calcific amorphous tumor: the CAT had a kitten. Cardiovasc Pathol 2007;16:115-8. 